\title{
A Novel Approach to Rank Text-based Essays using Pagerank Method Towards Student's Motivational Element
}

\author{
M Zainal Arifin ${ }^{1}$, Naim Che Pee ${ }^{2}$, Nanna Suryana Herman ${ }^{3}$ \\ Department of Electrical Engineering, State University of Malang, Indonesia ${ }^{1}$ \\ Faculty of Information \& Communication Technology, Universiti Teknikal Malaysia Melaka, Malaysia ${ }^{1,2,3}$
}

\begin{abstract}
Learning outcomes is one of the important factors to measure student achievement during the learning process. Today's learning is more focused on problem-solving and reasoning to existing problems than an ordinary problem. Most exams have been directed to analysis questions for students to think and synthesize. As such is troublesome for most students, they are not ready to answer the question, thus, their answers almost similar to their friends. This implies that the teacher has tried to guide students to work professionally and originally. However, the Teacher facing difficulty assessing student's work, especially if the assignments are conducted online without faceto-face instructions/discussions. To bridge such a gap, the teacher needs a method or algorithm to measure their rank to encourage students making an original answer. This research provides a solution in calculating students' ranks based on the similarity score of the essay answers. Pagerank is a ranking algorithm used by Google, this algorithm utilizes a Markov matrix that contains the direction of similarity score for each student. These scores are computed by the power method until converging. Rank is displayed to the teacher to review the similarity level of students' answers. As such is presented a line chart in which the $x$-axis refers to the students and the $y$-axis depicts the level of similarity. Ranking computation in Matlab produces an Eigen vector which acts as the rank measure. The higher the rank, the more similar is their answers to others. Hence, students with high ranks to work their answers more seriously ensure their original thoughts. In conclusion, the similarity score matrix using the PageRank algorithm can contribute to the teacher in providing peer motivation and encouraging student's internal motivation by presenting the ranked-answers presentation.
\end{abstract}

Keywords-Pagerank; learning outcomes; similarity

\section{INTRODUCTION}

Online Learning becomes the requirement in industrial era 4.0 to gain the easiest and fastest way of the learning process based on technological development. Teachers need it to get the student's exam results promptly and manageable in digital format. An essay test is still the best method to assess a student's analytical ability to the given problem. The students to practice his/her skills to analyze and synthesize, as a way to develop their knowledge.

In the modern learning goal, a teacher administers an exam at the end of the semester and she or he reviews those exams accordingly, often the teacher finds it difficult to identify the originality of student response and especially their similarity score. Teachers need an alternative method to measure student's scores and their ranks. Thus one can anticipate to whom student needs extra motivation during the learning process.

E-learning application provides similarity checking on essay responses. Unfortunately, as such is not quite informative for the teacher such as an unknown source of students copied work and a similarity relationship between one another. Based on the observations conducted for about a month on several campuses in Indonesia, teachers claim that motivation is compulsory for students, by presenting similarity scores as a visual report, i.e. graph, chart or diagram.

PageRank is a ranking algorithm used by Google to calculate web page rank based on a directed relationship of hyperlinks among others. It uses a power method formula which calculates a Markov matrix to produce Eigen vector. An Eigen value is related to its convergences, As a result, Google named it PageRank. PageRank computations take several hours, depends on the Markov matrix size, to reach convergence with initial Eigen vector related to Markov matrix character.

This research is important to do because lecturers find it difficult to motivate students based on their work as has been investigated by [1], besides that research objective focuses on developing a relationship between students based on the similarity score test using the PageRank method. It consists of parameters: including similarity score and student location (latitude and longitude). Meanwhile, the research scopes are: 1) exams take place is an essay type, and 2) the student's location is based on the current local address detected by GPS or browser with location plugin support.

\section{LITERATURE REVIEW}

In this section we cover some foundations that grounds this research consisting of student ranking, plagiarism among students, students' motivation and page rank method.

\section{A. Student Ranking}

Vieira (2015) claims the superiority of the strategic value is given by the academic ranking process as globally and influences academic quality in scientific activities c. This article accentuates that student rank has a positive impact to the quality and learning process. Unfortunately, the author does not mentions the cause and effect in a case where a student was ranked at the low level. As such should lead to the strategic action to be taken, in which the paper is also lacking of. 
Furthermore, Keller (2016) explains that in order to improvinge students' intelligence affective factor takes control, the teacher must have both knowledge and the right strategy to motivate students [2]. Affective intelligence and intellectual intelligence are inseparable to ensure students achieving good learning outcomes.

Surveys carried out by Buckley (2016) reveals that gamified learning has a good impact for the student's learning process. Rosyid (2018) also reveals that game-based learning strategy can creates a fun-learning environment. However, it is inevitable that participation may varies depending on intrinsic or extrinsic motivation of the student concerned. The results of these study are interesting to analyse by educators, the fact that students at any level of education can take part in such learning methods[3]. But it is not discussed if students dislike a learning material, to which strategy the teacher must apply to present the motivation.

Marks (2017) said that education process which includes the effectiveness of learning will affects the quality of learning and increases learning achievement by factors such as course recommendations, student skills, and behavior detection [4].

Denning (2018) said students with the top three academic rank, potentially obtains high test score in the coming exams. Even, they are found to be easily enrolled the college level. This is influenced by the quality and ranking of the school [5]. Students in the higher-ranked schools have higher average exam score than students from lower-ranked schools.

However, Murphy (2018) explained that the potential benefits of schools that have high rankings will be influenced by the confidence ranking of students who are poorly ranked. With the influence of this ranking, investment in education will decline [6]. Meanwhile, student self-motivation is still a new research area. Unfortunately, the author did not explain that good school rank is influenced by students rank well. And there is no relationship between low-ranked students and school's rank. In facts, there are other factors, involved as well such as school's accreditation.

\section{B. Plagiarisme Over Students}

According to $\mathrm{Hu}$ (2015), rules that are made based on detection and focus on giving penalties are more effectively applied to Chinese students. On the contrary, based on other findings, it shows that an education-based approach that motivates them is better than giving a punishment [7].

Cronan (2018) mentions behavior patterns, morals, attitudes are the biggest factors that influence a person to academic integrity especially when not to do plagiarism and share homework. The author gives an overview of $33 \%$ and $35 \%$ of students working on academic task do plagiarism or sharing homework [8]. The author did not clearly state what type of motivation had been given to students and how many students did plagiarism at the school.

Ehrich (2016) conducted research on 131 Australian students and 173 Chinese students to compare policies against plagiarism, there was no difference in plagiarism but rather understanding and plagiarism behavior [9]. There is no discussion in the paper about the response of students when the teacher motivated them regarding this plagiarism, but the results of this study were quite clear.

Khlifi (2017) conducted a simulations that the increasing quality of the learning process can be achieved by doing authentication security during the task process, so the possibility of plagiarism is minimal [10].

Sprajc (2017) in this study states that plagiarism is currently using technology that facilitates copy-paste and is transferred with students with low motivation to study well. Such a lacking motivation is not only due to plagiarism, but can be the product of a poor teaching method. Another research finds that students who spend time on the internet has no relationships to plagiarism scores [11]. Technology is a tool for facilitating goals, but plagiarism is more about self-awareness. Therefore technology should be the driving force for students to learn, where they can find sources of knowledge such as from the internet.

Kashian (2015) discusses that plagiarism is an ethical act carried out by students. This happens even though the teacher has provided advice and guidance to not perform such an action. But students, mostly, kept doing it. The results of this study indicate that students with awareness about plagiarism have a low plagiarism score. A teacher can help students to avoid plagiarism by using Turnitin application to check the results of his work. If there is found plagiarism, students are expected to act accordingly, for instance rephrasing the statements in his own words [12]. In some countries this method is not necessarily applicable, due to application licenses and fees. Some developing countries built their own plagiarism detection system and fully supported by the government.

$\mathrm{Ba}$ (2016) conducts research on 681 student articles at one of the universities in Vietnam using Turnitin and found that the level of plagiarism in that campus was higher than outside Vietnam with a percentage of $29.06 \%$. Plagiarism will lead to low academic value. In contrast, it will produce a positive effect when done honestly, although this task can take some times. So, it can be concluded that involving a plagiarism detection tool is very helpful in developing campus policies related to this [13]. In addition, the study did not explain that the acquired plagiarism included self-plagiarism or not. This is because when students writing articles, they sometimes copypaste statements from another section of the same article.

\section{Students Motivation}

Gianna (2017) provides an interesting picture that the teachers' motivation is actually contagious to their students. On the other hand, a teachers who is lacking teaching motivation can still improve students achievement [14]. This finding should enforce schools to encourage teachers teaching motivations. As such is beneficial to the school and the people involved.

Samir (2014) said that teachers need to understand the students' motivation when teaching online and it is very difficult to get because the interaction is not face-to-face. Teachers need to use another strategies including demographic variables and the use of technology to prepare the 21 st century generation [15]. 
Darwis (2016) study conducted on EFL fall students during the 2015/2016 semester shows that, plagiarism conducted by students was caused by the overly-difficult task material and students desire to graduate soon. Another cause is the openness of information stream where students can easily retrieve contents from the internet [16]. One could prohibit the use of internet-connected devices in class. However, it is all driven by the student's motivation in learning and his self-awareness.

Harits (2018) proposed approach in developing a SEG game, named Chem Dungeon, as a case study in order to demonstrate the effectiveness of media. This research making a good contribution to motivate the student via game learning [17].

Rocher (2018) reflects cognitive factor as the important aspect for a teacher to control his students, which including student involvement in learning, assignments and others. Plagiarism is a concern that students are not aware of. The paper emphasize on in class learning strategy where the teaching learning process becomes active in motivating students to avoid plagiarism [18]. However, the author fails to affirms that the easiest step is to announce plagiarism via a banner.

Johnson (2018) finds that plagiarism is a complex problem in a human state neither he is conscious or not, It is compulsory to seriously prevent plagiarism in order to maintain academic integrity on campus rather than penalizing plagiarists [19]. Johnson however, does not assert the prevention properly and the method to motivate students to get their hands off plagiarism.

\section{PageRank}

Amjad (2018) models an academic object ranking, including articles, journals, and conference proceedings. This ranking is based on the method of link analysis based on publication, number of citations, author's position, influence of the co-author, and topics in the scientific work. Each parameter influences rank scores to some degrees [20]. Link analysis is the basis of the page rank algorithm that uses the markov matrix. The weight of each citation is calculated based on the count and the list of references.

Massucci (2019) use pagerank algorithms to study scientific articles in a campus. Researchers believe that increasing a rank scores can be done by increasing academic ranking scores, such as via conducting internal or external citations between campuses, and the geographical location of the campus [21]. However, internal citation is susceptible to self-plagiarism that can drop the campus rank. A better alternative is conducting campus-to-campus citations and collaborates in the research community.

Gao (2016) explain that Pagerank index is the reflection of the popularity of publications, in the form of scientific works based on the article collection database. The author states that pagerank index is more suitable to use and is the main prediction parallel to h-index [22]. The h-index value is also influenced by the number of articles that Google recognize.

$\mathrm{Hu}$ (2015) applies pagerank algorithm to determine the referee in a match. It uses sensitivity analysis and general analysis methods in football matches, basketball, and baseball in determining coaches improvement analysis method for better result [23]. In addition such a method should fit for finding the best trainer for Olympics athletes or the likes.

Nykl (2015) aims to rank authors based on journal impact value using PageRank algorithm sourced from citation tracking. The result of this calculation is used to reward the author for the former year successful inclusion in the database. This algorithm solely use pagerank and the number of publications by eliminating self-citation [24].

Pagerank, which was developed by Google, has been used in various fields such as biology, computer science, physics, ecology, chemistry, sports and medicine to find ranks according to the research objectives. In addition, understanding pagerank charts makes it easy to visually understand the target set of interest [25]. PageRank algorithm selection is based on previous research which gives the fact that this algorithm is suitable for searching ranking based on direction or contribution from other nodes.

Let $A$ is adjacent matrix for graph with vertex $V=$ $\{1, \ldots, n\}$ and the graph is directed graph which $A_{i, j}$ is probability value of weight $(i, j)$. Suppose that $x$ is Eigen vector, $\lambda$ is Eigen value related to $x$, then power method denote as :

$A x=\lambda x$

Iterate that formula until convergence.

\section{MethodOLOGY}

In this research, we apply several procedures elaborated in the following section.

In general, out method refers to causal research where the problem has been clearly defined in the beginning. The main problem is how to calculate student ranking based on similarity score using power methods accurately.

Based on Fig. 1, there are several steps to follow (1) preparing dataset, (2) conducting primary data, (3) data analysis, (4) running power method and (5) ranks report. The following subsections elaborate each step in details.

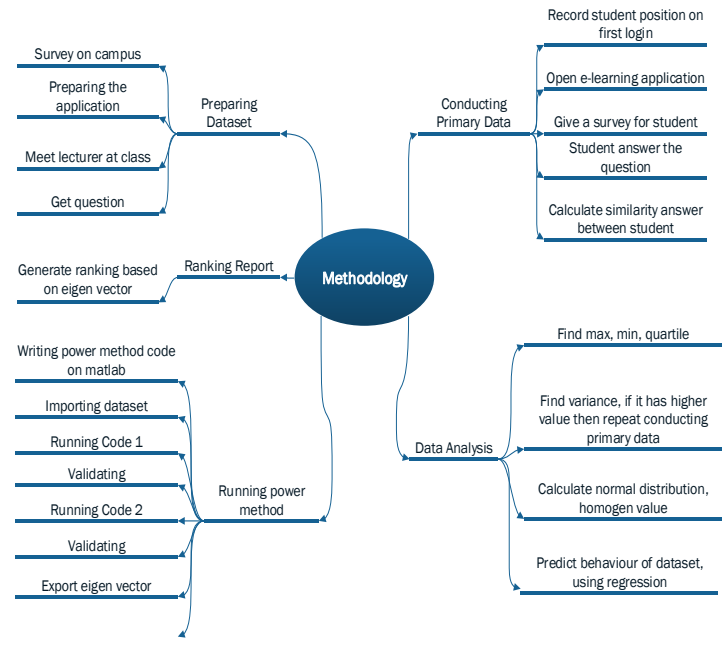

Fig. 1. Research Methodology (Clockwise Direction). 


\section{A. Dataset Preparation}

Dataset is the key factor of research, without a valid dataset the research is useless. There are two types of datasets, primary data and secondary data. Secondary data is to use existing data, while primary data is to collect data directly as conducted by this research. Preparing for primary dataset takes several steps.

1) Preparing the application: The e-learning application at UM campus has been developed since 2016. It focused on developing essays and the plagiarism detection application for used in student assignment assessment. Researchers collaborate with laboratory assistants in the Department of Electrical Engineering to prepare the applications prior to research. The application outputs students responses, similarity scores between students, student locations coordinates while doing the assesment (GPS latitude and longitude), response time for each answer, the timestamp when an answer is stored in the database table and the timestamp the response are submitted to the system.

2) Meet the teacher: The database course used as the experimental sample consisted of three classes, namely classes A, B, C, and D for each class containing approximately 40 students. Lecturer in this course, Ms Sophie gave permission to conduct research in his class on January 21, 2019. The lecturer said that students work on assignments online from their respective locations and some students work together in a certain location.

3) Course question: Questions are arranged in accordance with the material and syllabus in the course. The questions are as follows:

a) According to your understanding, how do you implement Hadoop on an e-commerce site? Describe your answers in detail starting from requirements engineering to reporting.

b) On the Hadoop platform there is a term map reduce that is used as a resource router on the Hadoop platform. Describe in details how the algorithm works.

c) Describe in details according to your understanding of the application of a relational database in the banking system where a good level of security and indexing is needed.

The questions take part in the e-learning database and will be displayed to students through an online learning system.

\section{B. Conducting Primary Data}

Primary data retrieval is carried out by researchers collaborating with lecturers at the UM campus. Some steps for taking primary data are as follows:

1) Record student position: Firstly, When students log in to e-learning system, they are required to enter a username and password, when they enter data correctly then the system will track the GPS coordinates in real, as well as the time login into the system.

2) Application start: After students have successfully logged into the system, then students take the exam accordingly with the schedule. Students work in two hours and they can do it anywhere.
3) Student answer the question: When students responds to exam questions, the system automatically save student answers every 5 minute, then, this answer will be stored in the server with including student answers, answering duration, and submission timestamp.

4) Student participate a survey: After student completed the exam, the students complete some items in a survey. This questionnaire contains questions related to this research including similarity answer, ranks and learning outcomes.

5) Similarity computation: Student answers in the database are then calculated using a formula developed by the researcher to product a similarity score.It has a unit of percentage from 0-100 and each answer has a similarity score except the uncomplated one. These results are then stored in the database for students to find out their similarity scores.

\section{Data Analysis}

We apply data analysis technique to measure the accuracy of a result. If the results of the analysis are reliable, the algorithm used is said to be efficient. Otherwise if the results of the analysis are not good, then the primary data retrieval is repeated.

1) Simple statistic requirement: The simplest analysis in looking at plagiarism scores is to use the minimum value, mode, mean, maximum value. The mean is used to determine the average plagiarism scores.

2) Homogen and variance: Ensuring homogeneous distribution is useful to see how the data is against other data, this is also aided by variance analysis that assesses each data to the average distance of all data. If the variance is small, then the data is spread evenly.

3) Normal distribution: Normal distribution is used to see the distribution of data at $5 \%$ beginning and end. Data that is normally distributed has a curve shape like a bell.

4) Regression analysis: The final part of this data analysis stage is knowing the behavior of the data using regression. The type of regression used depends on the characteristics of the data which will determine the trend of data based on other variables.

\section{Running the Pagerank}

The pagerank algorithm developed by Google adopted the power method formula by iterating the matrix Markov to converge. The calculation results are the Eigen vector and Eigen value values.

1) Writing Matlab code: Writing the Matlab code for power methods is actually not too complex, from Fig. 2 can be seen that there are some things that need to be considered so that the code matches the power method algorithm.

2) Importing dataset: In implementing this power method code, the input is the matrix markov where the data is taken from the student plagiarism score. as Fig. 3 describes direction factor of markov. The import dataset can be done by typing the command: $\mathrm{A}=$ loadMatrix; 
3) Running code: After the dataset is uploaded into Matlab memory, the next step is to run the Matlab code. The following example on Fig. 4 runs the Matlab code.

4) Validating: After the code is run, the next step is to validate the results of the calculation of the power method in the form of Eigen values and Eigen vectors. Validation is done using SPSS.

5) Export Eigen vector: In ranking calculation, the calculation results in Fig. 5 from the form of Eigen vectors stored in txt extension files. The example file is as follows.

6) Export Eigen value: The output of this calculation other than the Eigen vector also produces an Eigen value. This Eigen value is symbolized by $\lambda$. As an example, $\lambda=$ 0.0000438 .

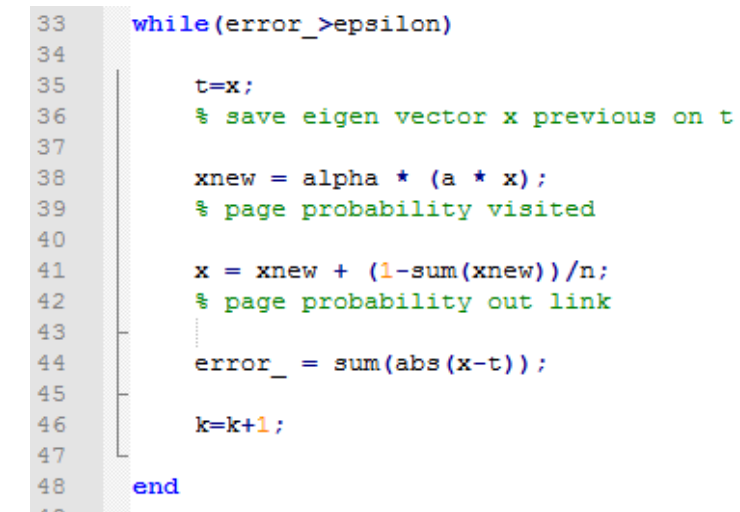

Fig. 2. Power Method Code on Matlab.

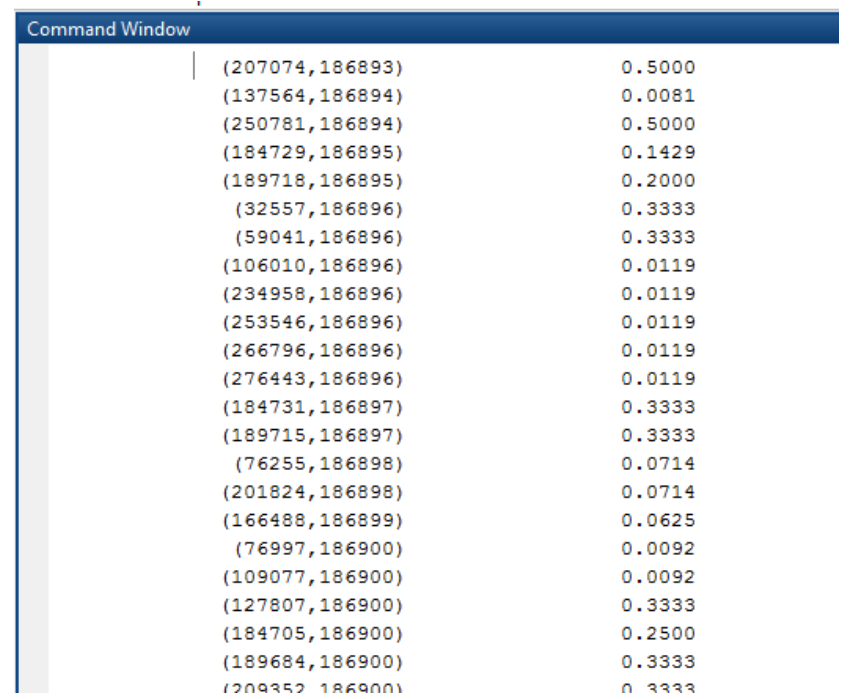

Fig. 3. Example of Process on Importing Dataset into Matlab Memory.

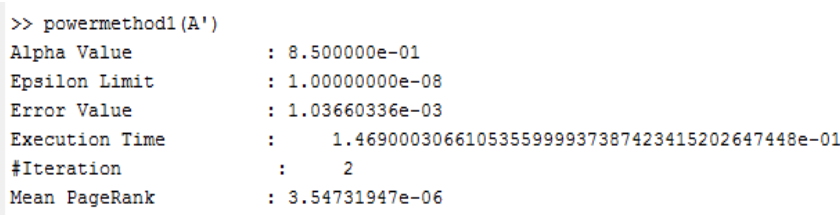

Fig. 4. Result of Power Method Calculation.

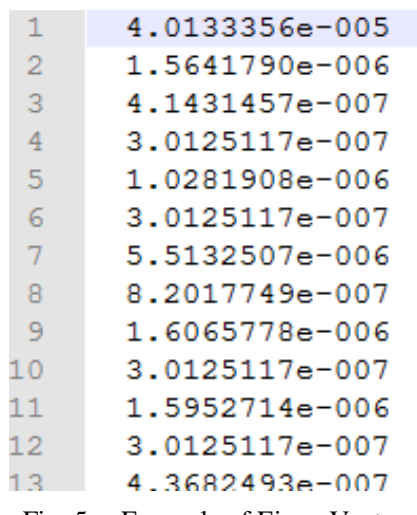

Fig. 5. Example of Eigen Vector.

\section{E. Ranking Report}

After the Eigen vector has been generated, then the next is the association based on the order of the student plagiarism score, the way to add it is in the database by importing the Eigen vector to the corresponding table. After that the results can be displayed in the e-learning application.

\section{RESUlT AND DISCUSSION}

This research provides several research results which will be discussed in this chapter. In addition to the results of the study, it is also discussed about the analysis of each outcome.

\section{A. Primary Data Acquisition}

Retrieval of primary data through several steps that begin from the preparation of the application, enter the question, retrieve the answer, until the results of the plagiarism score.

\section{B. Importing Question into Database}

In this study, the lecturer made a question for the test database subject as Fig. 6, while the questions were as follows.

a) According to your understanding, how do you implement Hadoop to e-commerce site? Describe your answers in detail starting from requirements engineering to reporting.

b) On the Hadoop platform there is the term map reduce that is used as a resource router on the Hadoop platform. Describe in detail how the algorithm works.

c) Explain in detail according to your understanding of the application of a relational database in the banking system where a good level of security and indexing is needed. table.

These questions are entered in the database in the question

After the questions are entered in the database, the questions can be used by the e-learning system. The online test is conducted on January 14, 2019 with a duration of 2 hours.

\section{Student Answer Result}

The online examination process has been carried out and runs smoothly, after students work on the exam questions then the next is to see the complete answers in the database even though students are not allowed to send blank answers, but need to be checked manually. 


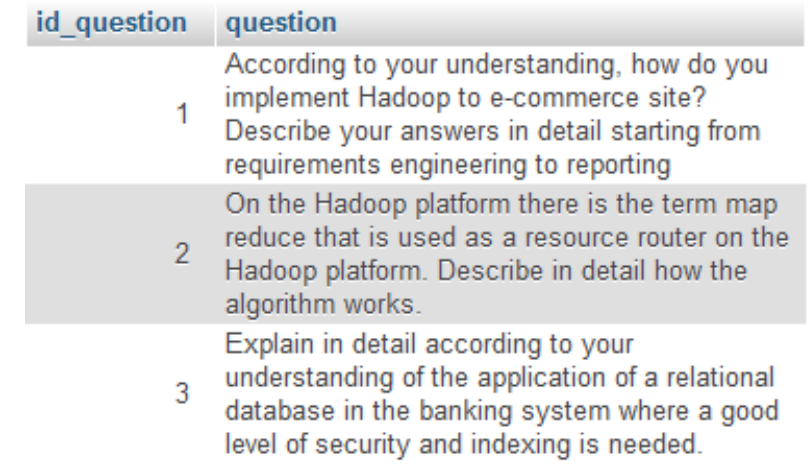

Fig. 6. Question Already Inserted in Database.

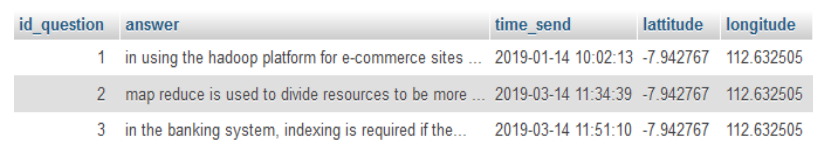

Fig. 7. Student Response Captured.

In Fig. 7, it can be seen that the system records the time of sending the answer, latitude and longitude, besides it also stores student information, subjects and other attributes.

\section{Execute Plagiarism Detection}

When the test time has been completed, the student cannot work again and the system will be closed, the answer at that time will be automatically saved in the database. Plagiarism calculations are then carried out automatically by the system, so researchers wait until the calculation is complete because this calculation runs cron jobs at midnight to save server load. The results of the calculation appear in Fig. 8.

At Fig. 8 it appears that plagiarism is stored in the plag_direction field as example 4 (43), 9 (10) means that the student has the same plagiarism as the student with id 4 of $43 \%$ and has plagiarism with students id 9 with a plagiarism score of $10 \%$.

\section{E. Transforming to Directed Graph}

When plagiarism has been obtained along with the direction of the sensitivity, then the next step is to change to graph form which facilitates analysis. The calculated similarity direction is the exit direction; the similarity of the dangling node type is ignored. Based on the number of students of 120 people, the number of graphs produced is 120 graphs. In Fig. 9, is given a brief explanation of the calculation results in the direction of graph.

\section{F. Generating Markov Matrix}

After all graphs are depicted, the next step is to change into the markov matrix. This markov matrix will then be processed using the power method algorithm. How to get matrix markov elements are as follows in Fig. 10.

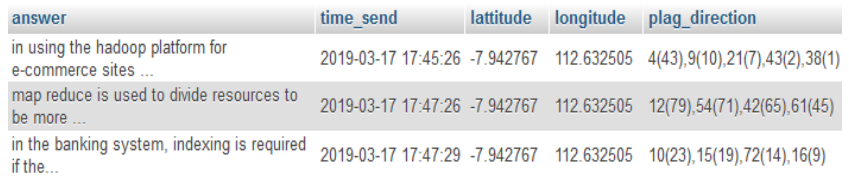

Fig. 8. Plagiarism Detection Result on Right Table.

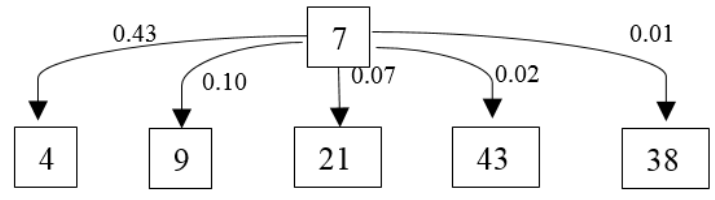

Fig. 9. Directed Graph.

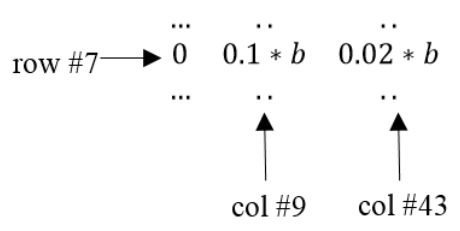

Fig. 10. Probability if Each Node.

From node 7 to node 4, it has a plagiarism score level of 0.43 , while node 7 has similarities in answers with nodes 4,9 , $21,43,38$. The number of inlinks to node 7 is 16 nodes, then the probability value in the markov matrix element is as with the value $b$ is the weight of node 7 for the inlink and outlink probabilities.

\section{G. Running Power Method}

This part is the part that determines the Eigen value and vector Eigen, before running the power method first write the code in Matlab. The code can be seen in Fig. 11.

\section{H. Eigen Vector}

After the calculation using power method is complete, Eigen values and Eigen vectors will be produced as show on Fig. 12. Eigen vectors are $n x l$-size vectors where each row is the ranking value of each student.

\section{Student Ranking}

After the pagerank calculation is complete and the Eigen vector is generated, the next step is to save the data into the database in the remaining answer table as Fig. 13. The elements in the Eigen vectors will be stored and then sorted by the ranking of the Eigen vectors that have been stored.

The greater the Eigen value, the greater the similarity level so that the ranking displayed is a sequence on the Eigen vector element which is reversed from low to high.

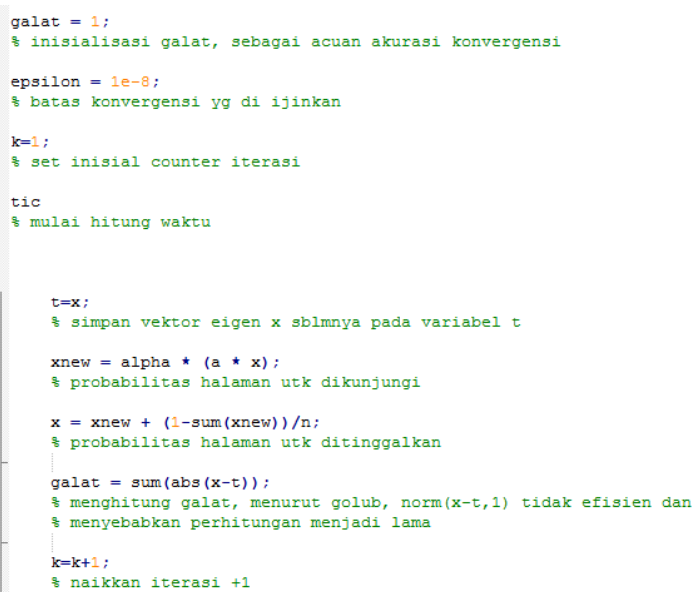

Fig. 11. Power Method Code on Matlab. 


\begin{tabular}{l|l}
\hline 64 & $1.7174761 \mathrm{e}-006$ \\
65 & $2.7294319 \mathrm{e}-007$ \\
66 & $7.3309226 \mathrm{e}-006$ \\
67 & $2.7294319 \mathrm{e}-007$ \\
68 & $4.8247916 \mathrm{e}-007$ \\
69 & $2.7294319 \mathrm{e}-007$ \\
70 & $1.1114967 \mathrm{e}-006$ \\
71 & $1.0284348 \mathrm{e}-006$ \\
72 & $2.4991175 \mathrm{e}-007$ \\
73 & $2.4991175 \mathrm{e}-007$ \\
74 & $3.8600658 \mathrm{e}-007$
\end{tabular}

Fig. 12. Eigen Vector as Result of Power Method Computation.

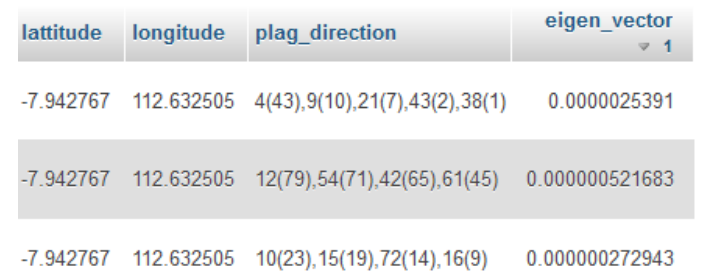

Fig. 13. Ranking Shows on the Right Hand.

\begin{tabular}{lr}
\hline studentName & eigen_vector $\Delta 1$ \\
\hline DANIAR WAHYU & 0.000000272943 \\
\hline MIFTAHUL ULUM & 0.000000521683 \\
\hline M. BADRUL HAQ & 0.0000025391
\end{tabular}

Fig. 14. Student Ranking Result.

In Fig. 14, it can be seen that students with the name DANIAR WAHYU have the first rank in the lowest plagiarism score, meaning that the student has a slight similarity, and is based on a rank value of $2.7 \mathrm{e}-7$. These students were attended by MIFTAHUL ULUM and M. BADRUL HAQ. Students who need to be motivated by the teacher are M. BADRUL HAQ, the teacher can provide motivation or together with other friends (peer motivation).

\section{CONCLUSION}

Student ranking is important for teachers to know students who need motivation to learn well. The ranking results are used by teachers in supporting learning but teachers often find it difficult to make rankings based on student work results. Teachers often give online examinations through e-learning sites and assess one by one the test results and then make a ranking.

PageRank algorithm that is used by Google gives the best ranking on Google search engines by providing document citations as a determinant of ranking. PageRank algorithm with a power method base is applied in ranking students based on similarity answers to the essay exam. Similar answers are a subset of other answers that are more complex and ranking calculations are done using Matlab.
The results of the calculations carried out provide vector Eigen results as the basis for determining ranking. This research provides a very useful implication that teachers are facilitated in determining groups of students with the same level of learning motivation so that teachers can provide appropriate advice. Teachers feel the ease in providing motivation to students because the teacher will definitely know which students will get more attention to motivate learning so that their learning outcomes increase.

Further research can be done by speeding up the iteration time on the power method, so the calculation time of iteration until convergent will be efficient.

\section{ACKNOWLEDGMENT}

I would like to thanks to Universiti Teknikal Malaysia Melaka, Universitas Negeri Malang for providing Laboratory assistance for PageRank computing.

\section{REFERENCES}

[1] R. Vieira, M. L.-H. E. Studies, and undefined 2015, “Academic Ranking-From Its Genesis to Its International Expansion.," ERIC.

[2] M. M. Keller, K. Neumann, and H. E. Fischer, "The impact of physics teachers' pedagogical content knowledge and motivation on students' achievement and interest," J. Res. Sci. Teach., vol. 54, no. 5, pp. 586614, May 2017.

[3] P. Buckley, E. D.-I. L. Environments, and undefined 2016, "Gamification and student motivation," Taylor Fr.

[4] A. Marks, M. Al-Ali, M. Majdalawieh, and A. Bani-Hani, "Improving academic decision-making through course evaluation technology," Int. J. Emerg. Technol. Learn., 2017.

[5] J. Denning, R. Murphy, and F. Weinhardt, "Class Rank and Long-Run Outcomes," 2018.

[6] R. Murphy and F. Weinhardt, "Top of the class: The importance of ordinal rank," 2018.

[7] G. Hu, J. L.-E. \& Behavior, and undefined 2015, "Chinese university students' perceptions of plagiarism," Taylor Fr.

[8] T. Cronan, J. Mullins, D. D.-J. of B. Ethics, and undefined 2018, "Further understanding factors that explain freshman business students' academic integrity intention and behavior: Plagiarism and sharing homework," Springer.

[9] J. Ehrich, S. J. Howard, C. Mu, and S. Bokosmaty, "A comparison of Chinese and Australian university students' attitudes towards plagiarism," Stud. High. Educ., vol. 41, no. 2, pp. 231-246, Feb. 2016.

[10] Y. Khlifi and H. A. El-Sabagh, "A novel authentication scheme for Eassessments based on student behavior over E-learning platform," Int. J. Emerg. Technol. Learn., 2017.

[11] P. Šprajc, M. Urh, J. Jerebic, D. Trivan, E. J.- Organizacija, and undefined 2017, "Reasons for plagiarism in higher education," degruyter.com.

[12] N. Kashian, S. Cruz, J. Jang, K. S.-J. of A. Ethics, and undefined 2015, "Evaluation of an instructional activity to reduce plagiarism in the communication classroom," Springer.

[13] K. Do Ba et al., "Student plagiarism in higher education in Vietnam: an empirical study," High. Educ. Res. Dev., vol. 36, no. 5, pp. 934-946, Jul. 2017.

[14] B. Gianna, R. Claudio, S. P.-P. economica, and undefined 2017, "Teacher Motivation and Student Learning," ideas.repec.org. 
[15] M. Samir Abou El-Seoud, I. A. T. F. Taj-Eddin, N. Seddiek, M. M. ElKhouly, and A. Nosseir, "E-learning and students' motivation: A research study on the effect of e-learning on higher education," Int. J. Emerg. Technol. Learn., 2014.

[16] S. Al Darwish, A. S.-I. E. Studies, and undefined 2016, "Reasons for College Students to Plagiarize in EFL Writing: Students' Motivation to Pass.," ERIC.

[17] H. A. Rosyid, M. Palmerlee, and K. Chen, "Deploying learning materials to game content for serious education game development: A case study," Entertain. Comput., vol. 26, pp. 1-9, May 2018.

[18] A. R. du Rocher, "Active learning strategies and academic self-efficacy relate to both attentional control and attitudes towards plagiarism," Act. Learn. High. Educ., p. 146978741876551, Mar. 2018.

[19] E. Johnson, "Situational Cheating Assessment of Motivation (SCAM): A Model for Understanding Student Plagiarism," 2018.
[20] T. Amjad, A. Daud, and N. R. Aljohani, "Ranking authors in academic social networks: a survey," Libr. Hi Tech, vol. 36 , no. 1, pp. 97-128, Mar. 2018.

[21] F. Massucci, D. D.-J. of Informetrics, and U. 2019, "Measuring the academic reputation through citation networks via PageRank," Elsevier.

[22] C. Gao, Z. Wang, X. Li, Z. Zhang, W. Z.-P. one, and undefined 2016, "PR-index: using the h-index and PageRank for determining true impact," journals.plos.org.

[23] Z.-H. Hu, J.-X. Zhou, M.-J. Zhang, and Y. Zhao, "Methods for ranking college sports coaches based on data envelopment analysis and PageRank," Expert Syst., vol. 32, no. 6, pp. 652-673, Dec. 2015.

[24] M. Nykl, M. Campr, K. J.-J. of Informetrics, and U. 2015, "Author ranking based on personalized PageRank," Elsevier.

[25] D. G.-S. Review and undefined 2015, "PageRank beyond the Web," SIAM. 